Rabaska

Revue d'ethnologie de l'Amérique française

\title{
Le patrimoine textile au Québec. Une tradition du geste, de la parole et... de l'écrit
}

\section{Anne-Marie Poulin}

Volume 12, 2014

URI : https://id.erudit.org/iderudit/1026789ar

DOI : https://doi.org/10.7202/1026789ar

Aller au sommaire du numéro

Éditeur(s)

Société québécoise d'ethnologie

ISSN

1703-7433 (imprimé)

1916-7350 (numérique)

Découvrir la revue

Citer cet article

Poulin, A.-M. (2014). Le patrimoine textile au Québec. Une tradition du geste, de la parole et... de l'écrit. Rabaska, 12, 153-159. https://doi.org/10.7202/1026789ar d'utilisation que vous pouvez consulter en ligne. 


\title{
Le patrimoine textile au Québec Une tradition du geste, de la parole et... de l'écrit
}

\author{
AnNe-Marie Poulin \\ Association des tisserands du Québec
}

\section{Introduction}

En 2013, l'Association des tisserands du Québec lance sa première publication, un modeste répertoire d'ouvrages portant sur des arts textiles et son milieu, publiés et édités exclusivement au Québec. L'événement vise à marquer les 80 ans des deux premières publications dans ce domaine, parues en 1933 et préparées sous la direction d'Oscar-Alphonse Bériau (1883-1947) alors directeur de l'École des arts domestiques à Québec. Bien que la transmission des traditions textiles repose pour l'essentiel, sur le geste et la parole, l'écrit a pourtant joué un rôle très important. En font foi ces deux textes, dont le premier trace l'impact de l'écrit au sein des Cercles de fermières du Québec et le second élabore sur le contenu du répertoire d'œuvres réalisées depuis les huit dernières décennies.

\section{Les Cercles de fermières du Québec (CFQ)}

Un des marqueurs de la vitalité d'une association est, sans contredit, sa durée' Force est d'admettre que le parcours de l'Association des Cercles de fermières du Québec, centenaire en 2015, est exemplaire. Cette association, forte de plus de 33000 membres, mérite une reconnaissance certaine pour avoir survécu aux turbulences du $\mathrm{Xx}^{\mathrm{e}}$ siècle et à sa séparation d'avec le ministère de l'Agriculture (MAQ) qui l'a créé et soutenu pendant près de 75 ans et, aussi, pour être demeurée la plus grande association féminine au Québec en dépit d'une appellation qui fait sourire ${ }^{2}$. D'autre part, des recherches montrent

1. Le documentaire Fermières, réalisé par Annie Saint-Pierre en 2013, fait part de cette réalité. La cinéaste brosse un tableau actuel de l'Association à travers la vision et les activités de quatre membres, dont l'auteur. D'après Éric Moreault, journaliste, Le Soleil, samedi 14 avril 2014, p. A18 :« Le résultat est profondément humain mais il nous laisse sur notre appétit » (http://voir.ca/cinema/2014/04/16/anniest-pierre-fermieres-tissees-serrees).

2. Rapport annuel du Cercle de fermières du Québec 2013-2014, Éditions Les Cercles de fermières du Québec. p. 8. Parmi les 33615 membres répartis en 648 cercles locaux, $2 \%$ sont agricultrices ; Claire Chénard, Les Cercles de fermières. Une appellation tronquée, Université Laval, mémoire de 
qu'un des facteurs de cette longue durée repose sur l'intérêt continu et renouvelé pour l'artisanat et ce, en dépit des idéologies, de l'urbanisation et des modes. D'ailleurs, parmi les divers comités structurant les $\mathrm{CFQ}_{\mathrm{FQ}}$, celui des arts textiles semble être le mieux connu du grand public et le plus apprécié des membres ${ }^{3}$. Sachant que l'apprentissage des techniques d'arts textiles relève, pour l'essentiel, du geste et de la parole, et sachant qu'aucun volume ne s'y consacre au Québec avant les premières décennies du Xx ${ }^{\mathrm{e}}$ siècle, il y a lieu de se demander par qui, comment et sous quelle forme ces savoirs et savoir-faire sont parvenus aux membres des CFQ.

Reconnaissons d'abord que, bien avant des parutions officielles liées aux arts textiles, avant même la création des CFQ en 1915 par le MAQ, la transmission des techniques artisanales est assurée par la famille, les congrégations religieuses et, enfin, les écoles ménagères. La première, qui voit le jour à Roberval en 1882 grâce aux ursulines de Québec, se verra déclinée sous plusieurs approches et appellations au cours du $\mathrm{Xx}^{\mathrm{e}}$ siècle $\mathrm{e}^{4}$. Aussi, en l'absence de volumes sur l'enseignement des techniques, il semble que ce soit uniquement les notes de cours des religieuses enseignantes et les cahiers d'échantillons soigneusement préparés par chacune des élèves qui demeurent la principale source de références. Et ce, pendant plusieurs décennies. Par contre, il ne fait aucun doute que les nouvelles écoles vouées entièrement ou en partie à l'enseignement ménager ont créé un terreau fertile pour l'avènement et l'essor des CFQ. En effet, les cours abrégés donnés aux membres des CFQ par l'entremise du MAQ, prolongent sciemment l'enseignement reçu dans les écoles ménagères. Ce qui, dans le jargon actuel, s'apparente à la formation continue, à l'éducation des adultes ou encore à l'éducation populaire.

À la fondation des $\mathrm{CFQ}$, les cours sont partagés entre l'étude des techniques agricoles et celles liées à l'industrie domestique 5 . Alors que le volet agricole profite déjà de volumes et de brochures imprimés sur l'aviculture, l'apiculture, le potager et le jardinage, l'enseignement des techniques liées à l'industrie domestique, dont le tissage, la couture, le filage, la chapellerie, le tricot,

maîtrise, 1981, 194 p. Selon Chénard, l'objectif explicite de la survivance du mode agricole de production serait subordonné à celui, implicite, de la survivance de la famille traditionnelle de même que des rôles féminins qui y sont associés (p. 30-31). D'ailleurs, dès la fondation des cercles, il appert que [...] pour répondre à l'apparente finalité agricole du mouvement, le MAQ établit une règle de principe pour l'admission des membres qui se limite à la simple manifestation d'intérêt pour les " choses agricoles " (p. 23).

3. Le Collectif Clio, Micheline Dumont, Michèle Jean, Marie Lavigne, Jennifer Stoddart, L'Histoire des femmes au Québec depuis quatre siècles, Montréal, Les Quinze, éditeur, coll.« Idéelles », [1982], éd. revue et corrigée 1985, p. 418. Rose-Hélène Coulombe, « La Transmission des savoirs dans les arts textiles et l'artisanat ", Histoire-Québec, vol. 12, n 3, 2007, p. 35-43.

4. Nicole Thivierge, Histoire de l'enseignement ménager - familial au Québec 1882-1970, Québec, Institut de recherche sur la culture, 1982, (475 p.), p. 241 ; Claire Chénard, op.cit., p. 46.

5. Yvonne Rialland-Morissette, Le Passé conjugué au présent. Cercles de fermières, historique 1915-1980, Montréal, Éditions Pénélope inc., 1980, p. 43-44. 
etc., exige que chaque élève prenne des notes, prépare ses graphiques, ses dessins et ses échantillons. Il est heureux que des techniciens et techniciennes à l'époque aient encouragé les cercles à se constituer une bibliothèque de références, d'échanges et de prêts à l'aide des volumes disponibles et des manuscrits de cours. Éventuellement, s'y ajoutent des tapuscrits, des extraits de revues et, parfois, la feuille de chou du cercle local avec ses trucs et astuces sur des techniques apprivoisées. La conservation de ces écrits était d'autant plus importante qu'il faut attendre 1933 avant d'enrichir ces modestes bibliothèques avec deux publications « officielles » vouées aux arts textiles : Teinturerie domestique et Tissage domestique ${ }^{6}$. Préparés sous la direction d'Oscar-Alphonse Bériau, alors directeur de l'École des arts domestiques de Québec, les volumes sont édités par le MAQ à l'intention des écoles ménagères et des « tisseuses» des Cercles de fermières. Rappelons que ces publications paraissent plus de 50 ans après la fondation de l'école ménagère de Roberval et 18 ans après la création des CFQ. Par contre, en termes de documentation imprimée, les $\mathrm{CFQ}_{\mathrm{FQ}}$ profitent très tôt dans leur histoire, d'une revue qui alimente les maigres sources existantes.

En effet, lors de leur premier congrès au Parlement du Québec en 1919, l'assemblée des membres des $\mathrm{CFQ}$ demande et obtient du ministère qui les parraine une revue trimestrielle, La Bonne Fermière, qui paraît à compter de 1920 jusqu'en 19317. Remplacée par La Bonne Fermière et la bonne ménagère de 1931 à 1932, elle cesse de paraître entre 1933 à 1941 en raison de la crise économique. De 1941 à 1944, elle reparaît sous le titre Revue des fermières $^{8}$, est publiée de façon intermittente en 1944-1945 sur fond de crise de sabordage générée par le haut clergé du Québec ${ }^{9}$, prend racine à nouveau avec pour titre La Terre et le foyer, pendant 20 ans de 1945 à 1964, et, de 1965 à 1974, elle est connue sous le nom Terre et foyer ${ }^{10}$. Au cours de cette période, des sources indiquent qu'en 1965 l'édition est publiée à 80000 exemplaires et est expédiée au Canada anglais, aux États-Unis, en Europe

6. O.-A. [Oscar-Alphonse] Bériau, Teinturerie domestique, Québec, Ministère de l'Agriculture, 1933, 188 p. ; Réimpression, Montréal, Léméac, Série " Trésors du patrimoine québécois », 1980, 188 p. ; id., Tissage domestique, Québec, Ministère de l'Agriculture, 1933, 219 p. (réimpressions 1938, 1939, 1943).

7. La Bonne Fermière, Québec, Ministère de l'Agriculture, 1920-1931.

8. Revue des Fermières, Québec, Ministère de l'Agriculture, 1944-45.

9. Sur la crise de sabordage, voir entre autres : Denyse Baillargeon, Brève histoire des femmes au Québec, Montréal, Boréal, 2012, p. 177-178 ; Le Collectif Clio, op.cit., p. 376-378 ; Yolande Cohen, Femmes de parole. L'Histoire des Cercles de fermières du Québec 1915-1990, Montréal, Le Jour, 1990, p. 51-59 ; Yvonne Rialland-Morissette, op.cit., p. 139-145 ; Simone Monet-Chartrand, Pionnières québécoises et regroupements de femmes d'hier à aujourd'hui, Montréal, Éd. du Remue-ménage, 1990, p. 201-203 ; également «Des tensions avec l'Église catholique » : http://ameriquefrancaise.org/fr/ article-696/Cercles_de_Fermières_du_Quebec.

10. La Terre et le Foyer, Québec, Ministère de l'Agriculture et de la colonisation, 1945 -1964 ; Terre et foyer, ibid., 1965-1974. 
et en Afrique. Entre 1974 et 1990, la revue est publiée de façon autonome par les CFQ en collaboration avec les éditions Pénélope inc. et porte le titre succinct Fermières $^{11}$; de 1990 à 1995, elle est prise en charge par les CFQ qui la renomment $L^{\prime}$ Actuel ${ }^{12}$; depuis 1995, la revue s'intitule L'Actuelle et est publiée cinq fois l'an. Au tournant des années 1980, le tirage atteignait 78000 exemplaires ; actuellement, il est de 37 500. Dès le début, la revue constitue une source importante de communication et d'inspiration pour les membres des cercles. Des extraits de la revue colligés dans des cahiers montrent d'ailleurs tout l'intérêt porté aux diverses techniques d'artisanat. Si le manuscrit avantage l'individu ou le groupe restreint, la revue favorise, quant à elle, la démocratisation des connaissances. Tout comme les volumes qui s'imposent avec le temps et en deux temps : avant et après la tutelle du ministère de l'Agriculture.

L'essor des CFQ et des écoles ménagères entre 1930 et 1950 explique que l'un des deux premiers volumes d'Oscar-A. Bériau, Le Tissage domestique lancé en 1933, a dû être réimprimé en 1938 et 1939, et revu et augmenté en $1943^{13}$. Ce même volume, traduit en anglais en 1939 sous le titre Home Weaving, fait également l'objet d'une réimpression en $1947^{14}$. Un autre ouvrage fort apprécié, Le Métier à quatre lames, paraît en $1941^{15}$. Toutefois, il s'écoule plus de trente ans avant que le ministère fasse paraître Tissage en 1974, une compilation de patrons de tissage sans présentation aucune ${ }^{16}$. Il faut dire que les $\mathrm{CFQ}$, incorporés en 1968, commencent tranquillement à se prendre en main. De fait, le MAQ cesse de publier la revue destinée aux Cercles de fermières en 1974. L'Association prend alors la relève et, avec les éditions Pénélope, publie, entre autres ouvrages pour les $\mathrm{CFQ}$, La Courtepointe, une tradition (1979) et Invitation au tissage (1985). L'élan est donné ! Dix ans plus tard, les CFQ éditent Les Arts textiles, trésors du patrimoine, récidivant en 2003 avec Les Secrets de la courtepointe et en 2009 avec Les Secrets du tissage ${ }^{17}$.

11. Fermières, Laval, Éditions Pénélope inc., 1974-1990.

12. L'Actuel, Longueuil, Éd. Les Cercles de fermières du Québec, 1990-1995 ; L'Actuelle, Longueuil, Éd. Les CFQ, Éditeur associé Tadam, 1995.

13. O.-A. Bériau (Oscar-Alphonse), Tissage domestique, Québec, Ministère de l'Agriculture, 1938. 219 p.; O-A Bériau (Oscar-Alphonse), Tissage domestique, Gardenvale Québec, Institut des Arts industriels, 1939, 219 p. ; O-A Bériau (Oscar-Alphonse), Tissage domestique, L'Islet, Nilus Leclerc, Ministère de l'Agriculture du Québec, nouvelle édition revue et augmentée, 1943, 332 p.

14. O.-A. Bériau, Home Weaving, Gardenvale, Québec, The Institute of Industrial Arts, Department of Agriculture, 1939, 219 p. ; $2^{\text {nd }}$ edition, Québec, Department of Agriculture, Arts and Crafts of Gardenvale inc., 1947, $300 \mathrm{p}$.

15. O.-A. Bériau, Le Métier à quatre lames, Québec, Ministère de l'Agriculture, 1941, 236 p.

16. Tissage, Québec, Ministère de l'Agriculture du Québec, Service de l'information, 1974, 88 p.

17. Collectif, La Courtepointe, une tradition, Laval, éd. Pénélope inc. 1979, 48 p.; Invitation au tissage, éd. Pénélope inc. 1985, 109 p. ; Collectif, Les Arts textiles, trésors du patrimoine, Les Cercles de fermières du Québec, 1995, 411 p. ; Collectif, Les Secrets de la Courtepointe, Les Cercles de fermières 
Malgré l'incorporation des CFQ en 1968, la scission définitive d'avec le MAQ n'arrive que 20 ans plus tard avec le départ ou la relocalisation des techniciennes affectées au service des cercles. Jusque-là, celles-ci sélectionnent et confectionnent les pièces à présenter aux concours d'art textile, préparent les critères d'évaluation, donnent la formation pertinente aux membres des $\mathrm{CFQ}_{\mathrm{FQ}}$ dans 25 régions du Québec, diffusent une information succincte aux cercles et effectuent l'évaluation finale. En prenant le relais après 1988, la direction des CFQ forme ses membres aux tâches reliées aux concours et publie chaque année à tirage limité, un cahier intitulé Concours d'artisanat textile $C_{F Q}$. Pour les cercles locaux et les adeptes de concours, ce cahier est un incontournable. Lors des journées de formation sur les pièces de concours, il faut voir toute l'attention portée à la lecture à haute voix des critères, des termes et détails techniques et enfin de la grille d'évaluation ${ }^{18}$.

À l'évidence, les membres avides de perfectionnement ne sauraient plus se passer de cet imprimé : un imprimé qui témoigne de l'importance de l'écrit comme support dans la transmission du geste et de la parole, qui fait valoir le parcours depuis le modeste manuscrit de l'élève, le tapuscrit de la technicienne, la feuille de chou du cercle local, les volumes officiels, la revue L'Actuelle, jusqu'à la création récente de sites électroniques par des Fédérations régionales et des cercles locaux. L'imprimé rappelle également que toutes ces formes d'écrits participent à une tradition intimement liée à l'histoire des femmes du Québec. Allant des pionnières maitrisant l'ouvrage des dames, comme Marie Rollet et Marie Guyart, aux congrégations religieuses ayant conservé, consigné et transmis leurs savoirs en arts textiles pendant plus de quatre siècles et aux techniciennes, ces «filles du gouvernement » qui ont sillonné tout le Québec pour former la relève qui s'active à son tour au sein des Cercles de fermières du Québec. Les publications du dernier quart $\mathrm{du} \mathrm{Xx}^{\mathrm{e}}$ siècle témoignent en quelque sorte de l'apport de chacune depuis la Nouvelle-France au Québec actuel. Ceci dit, il semble bien qu'à l'Association des Cercles de fermières du Québec, la tradition du geste et de la parole, arrimée à l'écrit, est là pour y rester. Ce qui est de bon augure pour leur deuxième centenaire.

\section{L'Association des tisserands du Québec (АтQ)}

Le second volet de cette note de recherche élabore sur le contenu du répertoire

du Québec, 2003, 158 p. ; Collectif, Les Secrets du tissage, Les Cercles de fermières du Québec, 2009, 331 p. Parallèlement aux publications sur les arts textiles, parait la série de 5 volumes Qu'est-ce qu'on mange? Cette série est sans contredit le véritable succès de librairie des CFQ.

18. Collectif, Concours d'artisanat textile, Longueuil, Les Cercles de fermières du Québec, publication annuelle depuis 1993. Ce cahier de près de 100 pages renferme les critères et formulaires du concours, de même qu'une présentation détaillée de chacune des techniques à réaliser. 
publié en 2013 par l'Association des tisserands du Québec (АтQ) ${ }^{19}$. Une recherche, étalée sur dix-huit mois auprès des membres de l'ATQ et de contacts extérieurs, a permis de dresser un premier bilan de la situation.

Ce répertoire, inspiré par le $80^{\mathrm{e}}$ anniversaire des premiers écrits au Québec voués aux traditions du travail textile, a été lancé à l'occasion du $33^{\mathrm{e}}$ congrès de l'Association des tisserands du Québec (AтQ) tenu à Québec les 31 mai, $1^{\text {er }}$ et 2 juin 2013. Il répertorie huit décennies de publications québécoises sur le filage, le fléché, la teinture et le tissage basse-lisse préparées et éditées par des gens d' ${ }^{\prime} \mathrm{ici}^{20}$. En tête de la nomenclature, on retrouve Tissage domestique et Teinturerie domestique, parus en 1933 sous la direction d'Oscar-A. Bériau, alors directeur de l'École des arts domestiques à la ville de Québec ${ }^{21}$. Outre le recensement d'ouvrages, ce premier répertoire de l'ATQ vise à évoquer et à mieux faire connaître des personnes, des organismes et des œuvres méconnues dans le domaine, tout en répondant à la mission de l'ATQ, laquelle est « vouée à la connaissance et à la promotion du tissage sous toutes ses formes et de ses techniques afférentes ${ }^{22} »$.

Le répertoire comprend trois sections : des articles sur le développement du textile au Québec, un visuel d'œuvres témoins à partir des premières publications d'Oscar-A. Bériau et, enfin, le recensement d'ouvrages sur supports divers.

La première section présente un aperçu de la diversité des approches en formation et en développement textile au Québec. Monique Dumas, directrice retraitée de la Maison Routhier à Québec, trace la genèse de la formation en art textile et rappelle ces artisans et artisanes qui, au cours des ans, ont travaillé dans l'ombre pour stimuler l'apprentissage et le développement des techniques artisanales au Québec. Anne-Marie Poulin cerne l'impact de l'écrit comme support à la connaissance des arts textiles auprès des Cercles de fermières du Québec depuis 1915 à nos jours. Jean-Louis Bouchard, directeur du Centre de formation et de consultation en métiers d'art de Québec, fait voir le succès des écoles-ateliers que l'organisme a mis de l'avant avec les cégeps de Limoilou et du Vieux-Montréal pour former des artisans professionnels. Le créateur du concept et du réseau Économusée, Cyril Simard, retrace l'historique et le développement de cette institution inédite consacrée à la promotion du patrimoine, la conservation de la tradition et la transmission du

19. Anne-Marie Poulin et Antoinette Roy (dir), Le Développement du patrimoine textile au Québec 1933-2013, vol. 1, n 1, Québec, L'Association des Tisserands du Québec, 2013, 34 p.

20. Les quatre techniques retenues correspondent à la thématique générale du congrès. Faisant d'abord écho à une partie du cursus de l'École des arts domestiques des années 1930, elles ont été retenues comme sujet d'étude dans les ateliers du congrès 2013 et ont alimenté une exposition de livres rares, de documents divers et d'artefacts pour les congressistes et le grand public.

21. $C f$. notes 6 et 13 .

22. L'Association des tisserands du Québec, Statuts et règlements, 2011, article 4, p. 2. 
savoir de l'artisan. François Beauvais, directeur du Conseil des métiers d'art du Québec, rend hommage aux artistes, artisans et artisanes des métiers du textile dans l'expression contemporaine en métiers d'art, évoque le nom de certains parmi eux qui se sont illustrés et souligne le rôle de cet organisme conseil dans le développement des disciplines et des carrières. Sur une note plus personnelle, Agathe Collard fait l'éloge de son aïeule, lui permettant de situer le tissage dans la continuité ; Doreen Page de l'Estrie décrit son parcours en tissage à compter des années 1980, alors qu'une première recherche sur la formation des tisserandes de l'ouest de l'Île-de-Montréal, effectuée par AnneMarie Poulin, illustre bien la perméabilité entre les artisanes anglophones et francophones au Québec en dépit de la barrière de la langue.

La deuxième section, sous l'intitulé « Témoins de la mémoire textile 1933-2913 », occupe deux pages placées en vis-à-vis et présente un visuel de 24 pages couvertures parmi les quelque 80 publications parues depuis les premiers livres d'Oscar Bériau en 1933. La troisième section recense au-delà de 80 ouvrages portant sur le filage, le fléché, la teinture et le tissage basse-lisse. C'est sans surprise que le tissage compte le plus grand nombre de mentions, suivi du fléché, de la teinture et du filage. À noter que le fléché a même fait l'objet d'une thèse de doctorat en 1996 faisant habilement la preuve que cette technique, unique au Québec et au Canada français, est bel et bien un tissage au doigt et non un tressage. Un relevé de revues, de supports divers, de livres en préparation ou de lectures complémentaires boucle cette dernière section.

On ne peut certes prétendre à un état exhaustif des lieux du fait que le relevé s'est limité à quatre techniques. Toutefois, cette publication unique donne un aperçu de la dynamique de la tradition textile au Québec et illustre la complémentarité entre le geste, la parole et l'écrit dans l'acte de réalisation d'une œuvre. Le chercheur tout comme le praticien y trouve donc son compte. Vivement, que la recherche s'étende à d'autres formes d'arts textiles dans un avenir rapproché ! 